\title{
Measures against preschool noise and its adverse effects on the personnel: an intervention study
}

\author{
Fredrik Sjödin • Anders Kjellberg • Anders Knutsson • \\ Ulf Landström $\cdot$ Lennart Lindberg
}

Received: 21 May 2012/ Accepted: 22 November 2012/Published online: 27 December 2012

(C) Springer-Verlag Berlin Heidelberg 2012

\begin{abstract}
Purpose The aim of the study was to analyze the exposure effects of different types of noise measures carried out at preschools. The project was carried out as an intervention study.

Methods The investigation included 89 employees at 17 preschools in the northern part of Sweden. Individual noise recordings and recordings in dining rooms and play halls were made at two departments in each preschool. The adverse effects on the employees were analyzed with validated questionnaires and saliva cortisol samples. Evaluations were made before and 1 year after the first measurement. Between the two measurements, measures were taken to improve the sound environments at the preschools.

Results The effects of the measures varied a lot, with respect to both the sound environments and health. Regarding acoustical measures, significant changes were seen for some of the variables analyzed. For most of the tested effects, the changes, however, were very small and non-significant. The effects of organizational measures on the objective and subjective noise values were in overall less pronounced.

Conclusion Acoustical measures improved the subjectively rated sound environment more than organizational measures. This may be due to the high work effort needed
\end{abstract}

F. Sjödin $(\bowtie) \cdot$ A. Kjellberg · U. Landström · L. Lindberg Center for Built Environment, University of Gävle, 80176 Gävle, Sweden

e-mail: fredrik.sjodin@hig.se

\section{A. Knutsson}

Department of Public Health, Sweden Mid University,

85170 Sundsvall, Sweden to implement organizational measures. Even though the sound level was not lower, the personnel experienced improvements of the sound environment.

Keywords Noise - Measures - Stress - Health · Occupational environment $\cdot$ Intervention
Abbreviations
SOFI Swedish occupational fatigue inventory
SMBQ Shirom Melamed Burnout Questionnaire
ERI Effort-reward imbalance
KSS Karolinska Sleepiness Scale
KSD Karolinska sleep diary
MDI Major depression inventory
CAR Cortisol awakening response
CDD Cortisol decline over day

\section{Introduction}

Several physical characteristics make noise exposure in preschools problematic from the perspective of health hazards (Sjodin et al. 2012b). The noise levels are in the range of $60-80 \mathrm{dBA}$ and thus relatively high, considering the pedagogic work carried out. The complex and varying noise exposure, mostly a result of the children's activities, also increases the risk of stress and fatigue (Sjodin et al. 2012a). Masking and annoyance might under certain circumstances have strong negative impacts on education. Besides annoyance and masking, the exposure may increase the risk for hearing damages, tinnitus and sound fatigue.

The need of measures for improvements of the noise environment as well as the health status of employees has 
been announced from several authorities. According to the Swedish Work Environment Authority, the preschool of today is one of the work environments with the largest increase in reported noise-related ill health, stress and physiological distress (Swedish Work Environment Authority 2010).

Realistic measures directed upon the noise exposures in preschools differ in several aspects from a traditional approach. Traditionally, for example as applied in industrial environments, measures are prioritized in the following order: dampening the sound at the source, reducing its propagation and providing personal hearing protection (Snyder 2000). According to a number of recent studies, the noise exposure of the preschool personnel is dominated by the children's voices and playing activities (Sjodin et al. 2012b). Being part of the educational work, the possibilities to reduce the child-related sound generations with traditional technical methods are limited. To reduce the sound from these sources (Smith 1996; Hansen 2005; Bies and Hansen 2009), other physical and organizational methods must be used. Furthermore, measures focused on the receiver, that is, plugs or ear muffs, are in most cases an unrealistic approach. Reductions in the hearing capacity of the employees are in conflicts with the pedagogic work and looking over the children.

Noise reduction in preschools is thus a more complex problem than the traditional noise problems in work places. Being so, the noise situation at a preschool can seldom be solved by one single measure. Furthermore, a measure that is successful in one preschool is not necessarily the best alternative in other schools.

Measures tested in preschool environments can be categorized into acoustic and organizational alternatives. Acoustic measures include damping of the walls, damping of the floor, damping of the tables, quite toys, silent areas, acoustic control, lowering the number of children at the department, etc. The category of organizational measures can include organizing the play groups, education of the personnel and children, recovery possibilities, etc.

In the present study, we tested a number of measures aimed to improve the noise situation at the preschools and to improve the health of the employees. In each preschool, only one measure was tested. The effects of the measures were evaluated in terms of changes in noise exposures and also physiological and behavioral responses of the personnel.

In a previous study (Sjodin et al. 2012a), health issues of the preschool personnel were seen in terms of tinnitus, noise fatigue, stress and burnout. The personnel with the strongest ill health also reported that they were more annoyed by the noise at the places of work.

\section{Aims}

The aim of the intervention study was to analyze the exposure effects of different types of noise measures, especially to analyze the effects on experiences and health and to analyze the way in which active contribution from the personnel in the measures interfered with the effects of the measures.

\section{Methods}

Design of the intervention study

The study was carried out for 1 week at each preschool before and after different measures had been applied at the preschools. After the first data collection, the preventive measure was introduced at the preschool. The follow-up measurements were conducted 1 year after the first data collection period.

\section{Participants}

All preschools in the municipal area $(N=64)$ were invited through the local authorities. Seventeen schools voluntarily chose to participate in the study. In cooperation with the principal at each preschool, two departments per preschool were selected to participate. Departments with planned physical changes or many temporarily employees were excluded. At each department, three employees, working more than $30 \mathrm{~h}$ per week, were offered to participate in the study.

To make repeated within-subject analyses possible, employees only participating in both the first and the second study period were included in the study. In total, 77 females with a mean age of 42.3 years (SD 9.8 years) and 12 males with a mean age of 39.8 years (SD 11.0 years) participated in the study. In total, 89 subjects were included in the study with a mean age of 41.9 years (SD 9.9 years).

\section{Presence of children}

The presence of the children at the departments was registered by the participating employees at the departments. Age, gender and number of children for each day of the study week were registered in a standardized form.

\section{Noise exposure}

Noise exposures of the employees were analyzed through personal and stationary recordings before and after the implementation of the different measures. All participating personnel carried a noise dosimeter during all work hours 
of the investigated week. Two types of noise dosimeters were used: Brüel and Kjaer 4445 and Larson Davis 706-Atex. The participating employees made notes if there were any technical problems with the equipment.

The stationary recordings were carried out during all working days, using two Brüel and Kjaer 2260 investigators, Monday to Friday. Sound levels were measured in one dining room and one play hall at each department. They were placed at $2 \mathrm{~m}$ height in the center of the room. Analyzed noise exposure values consist of $\mathrm{dB}(\mathrm{A}), \mathrm{dB}(\mathrm{A})$ Max and fluctuations in the noise levels, in terms of number of sound events above $85 \mathrm{dBA}$ per hour. A deeper description of the recordings and analyses of the noise exposures is given in Sjodin et al. (2012b).

\section{Questionnaires}

Demographic data regarding the working environment, medication, personal health, age, gender, etc. were collected in a questionnaire provided to the participating employees.

Furthermore, on Wednesdays, the participants were asked to answer two questionnaires to measure stress/ energy and subjective fatigue at four different time points (at wake-up time, $1 \mathrm{~h}$ after wakeup, 11:00 am and 9:00 $\mathrm{pm})$. The questionnaires were answered after leaving a saliva sample for cortisol analyses (see below).

The stress-energy model was used to measure the subjective stress and energy and its relation to the psychosocial work conditions (Kjellberg and Wadman 2002). The questionnaire is based on twelve items designed to measure two factors: stress and energy. The scale for each item ranges between 0 and $5(0=$ not at all, $1=$ hardly any, $2=$ to a little degree, $3=$ to some degree, $4=$ to a high degree, $5=$ to a very high degree; Wadman and Kjellberg 2007; Eklöf et al. 2004; Persson et al. 2003; Norlander et al. 2005). A mean score of 2.4 was established as the neutral point of stress (Åhsberg et al. 1997; Åhsberg 2000).

Subjective fatigue was evaluated by use of the Swedish Occupational Fatigue Inventory method (SOFI; Åhsberg et al. 1997; Åhsberg 2000). The SOFI questionnaire was administered along with the stress-energy questionnaire. SOFI was designed to measure fatigue in five dimensions: lack of energy, physical exertion, physical discomfort, lack of motivation and sleepiness. Each dimension was measured using five items rated from 0 to $5(0=$ not at all, $1=$ hardly any, $2=$ to a little degree, $3=$ to some degree, $4=$ to a high degree, $5=$ to a very high degree). We choose to use the dimension "lack of energy" as the indicator of fatigue.

To evaluate burnout syndromes, the Shirom-Melamed Burnout Questionnaire (SMBQ; Melamed et al. 1992, 1999) was used. The SMBQ used four subscales with a total of 22 items. Each item is graded on a seven-point scale ranging from 1 to $7(1=$ almost never, $7=$ almost always). The defined subscales were the following: emotional and physical exhaustion, tension, listlessness and cognitive weariness. All subscales were calculated, and from the obtained score, the subject was characterized into one of four burnout groups (healthy $=0-2.75$, low burnout $2.76-3.75$, high burnout $=3.76-4.46$, pathologically burnt out 4.47 and higher; Grossi et al. 2003; Melamed et al. 1992; Shirom et al. 1997).

Employees rated effort at work and the reward they experienced according to the effort-reward imbalance model (ERI; Siegrist 1996). The ERI model measured the effort in the daily work in relation to the reward the employees received in terms of appreciation, feedback and material assets. A score based on the ratings was calculated. A value higher than 1.0 indicated a severe imbalance between effort and reward.

Sleep quality before and after the introduction of the different measures was evaluated using the Karolinska Sleepiness Scale (KSS) and the Karolinska Sleep Diary (KSD; Akerstedt et al. 1994). The subjects filled out the KSS and KSD questionnaires every morning during the work days, Monday to Friday.

KSS measures the alertness or sleepiness at time going to bed and at time of wakening, by the use of a nine-point scale from 1 to $9(1=$ very alert, $3=$ alert, $5=$ neither alert nor sleepy, $7=$ sleepy, but with no difficulty staying awake, and $9=$ very sleepy, fighting against sleep, requiring great effort to stay awake).

KSD measures several aspects of sleep quality such as number of wakeups, waking up too early, quality of sleep, dreams on a one-to-five scale with different verbal anchor points depending on the question.

Based on the KSD questionnaire, we created a sleep index regarding disturbed sleep. This index was the sum of the scored values for the questions: stress before going to sleep, difficulties falling asleep, trouble falling asleep, sleep quality, disturbed or restless sleep, premature awakening, time awake during the night, enough sleep, deep or light sleep, easy getting out of bed in the morning, fully rested. A low score was thus an indication of poorer sleep quality and recovery. Reliability analyses were made using Cronbach's alpha. The Cronbach's alpha score of the created index was 0.80 .

The Major Depression Inventory (MDI; Bech et al. 2001; Olsen et al. 2003) was used to evaluate depression among the employees. MDI is a widely used questionnaire where a total score is calculated by use of the WHO ICD-10 algorithms for depressive symptomatology and severity (WHO 1993). The MDI questionnaire used the last 2 weeks as a time frame. The subjects were asked to answer eleven questions on a scale from 1 to $6(1=$ all the 
time, 2 = most of the time, $3=$ about half the time, $4=$ less than half of the time, $5=$ small amount of the time, $6=$ at no time). A score between 0 and 50 was calculated which thereafter was used to classify the subjects into different groups of depression severity (Olsen et al. 2003).

All employees were asked to evaluate how well the introduced measure improved the sound environment at the department using a four-point graded question $(1=$ not at all, $2=$ to some degree, $3=$ to a high degree, $4=$ to $a$ very high degree).

\section{Saliva cortisol}

Saliva cortisol was collected by using saliva sampling kits (Salivette $^{\circledR}$, Nümbrecht Germany). All participants left four saliva samples during Wednesday (at wakeup, $1 \mathrm{~h}$ after wakeup, at $11 \mathrm{am}$ and at $9 \mathrm{pm}$ ). The participants were instructed to store the samples in a refrigerator at home and bring them to a refrigerator at the preschool as soon as possible. All samples were collected at the end of the study week and stored in a freezer $\left(-20^{\circ} \mathrm{C}\right)$ until analyzed at the Stockholm University Stress Clinic. Analyses were made according to Orion Diagnostica Spectria ${ }^{\circledR}$ Cortisol RIA (Orion 2010).

Besides using cortisol values from the different time points in the analyses, cortisol awakening response (CAR) and cortisol decline over the day (CDD) were calculated. The cortisol curve in blood is characterized by high levels in the morning and low levels in the late evening and beginning of the night. Upon waking, an increase of $50-100 \%$ is observed (Clow et al. 2004). In 15-25\% of subjects, CAR is not present (Wüst et al. 2000). The CAR is superimposed on the circadian variation of cortisol secretion. Some studies have shown that chronic stress is associated with increased CAR, but the findings are heterogeneous (Kudielka and Wust 2010). CDD is a measure of cortisol variability. Exhausted individuals have a low cortisol variability (Lindeberg et al. 2006).

\section{Preventive measures}

The measures tested at the participating preschools are described in the overview below. At the two departments of each preschool, only one measure was tested. Reducing the number of children was the only measure tested at two departments at two preschools. Only one measure was evaluated at each preschool to assure that the result could be interpreted as a result of specific measures. In order to analyze the measures on group basis, 13 measures were categorized into physical and organizational type of measures. The three preschools without any measure tested were assigned as control group. Some of the measures categorized as acoustical measures can be argued also to have an impact on organizational aspects of the work and vice versa. The aim of the categorization was to simplify the practical application of different types of measures. Besides, the categorization into acoustical and organizational measures also reflects the way in which the personnel were engaged in and contributed to the measures. Once being established, the contribution from the personnel in the use of acoustical measures was little or none. The organizational types of measures, on the contrary, were based on more or less continuous engagements and support from the personnel.

All preschools differ also in their construction, and some preschools already had some of the measures installed before the introduction of the project. Each measure is rather costly and time-consuming to implement, and an increase in a higher number of preschools and measures was not practically applicable. By introducing only one specific measure at one preschool, the potential effect of the measure might therefore be rather low, thus causing a low power.

\section{Acoustical measures}

Five of the tested measures are characterized as acoustical measures with an aim to improve the sound environment at the preschools either by lowering the equivalent sound level or by improving experienced sound quality.

\section{New ventilation system}

The ventilation system of the participating departments was rebuilt with a noise reduction, especially in the lowfrequency range. The background noise of the ventilation at the departments was tested before and after rebuilding the system using stationary recordings. On average, the dBA level of the ventilation noise was reduced from $31 \mathrm{dBA}$ (SD 7.14) to $28 \mathrm{dBA}$ (SD 3.16).

\section{New tables}

At this school, the tables were changed into dampened alternatives. The new tables (Balzar Beskow AB, Sweden) were constructed by use of noise isolation and with damping material on the surface of the table. The tables were constructed with the aim of dampening noise generation from objects in contact with the surface, such as forks, bricks during eating and also noise related to the use of toys.

\section{Noise isolation wall panels}

In this preschool, new noise-isolating wall panels (Wall panel $\mathrm{C} /$ Texona) were mounted on the walls of the dining 
rooms and playing rooms. The sheets were constructed by Saint-Gobain Ecophon AB, Sweden, with a sound absorption factor of $\alpha_{\mathrm{p}} 1.0$ in the frequency range of $500-1,000 \mathrm{~Hz}$ and about $\alpha_{\mathrm{p}} 0.9$ in the frequency range $1,000-4,000 \mathrm{~Hz}$ according to the SS-EN ISO 11654 classification.

\section{New toys}

The use of noisy toys was identified by the employees at the two departments and by the research group. On average, $10-15$ toys were considered as noisy in the perspective of generating high levels or pronounced transients and peaks. The unfavorable toys were changed into corresponding silent alternatives, for example by use of building blocks constructed with soft material instead of wood, rubber wheels instead of metallic or hard plastic wheels, ringing bells changed into light signals (Lekolar, Sweden).

\section{Reduced number of children}

A reduction in the number of children was administrated at two preschools. At each of the schools, the number of children was reduced by two children. At one preschool, in both departments, the number of children were reduced from 17 to 15 children, and at the other preschool, the number of children were reduced from 18 to 16 at one department and from 24 to 22 at the other department.

\section{New play hall}

Rebuilding of the play hall was carried out at one preschool to obtain alternatives for dividing the larger groups of children into smaller groups, especially during periods of playing. By rebuilding the preschool, in total four new spaces were obtained from one bigger room.

\section{Organizational measures}

Seven of the tested measures are characterized as organizational measures with an aim to improve the sound environment through changes in the work at the departments. The aim of these measures was to lower the sound level and improve the subjective experienced work load and stress.

\section{Recovery room for the children}

At two departments at one preschool, new rooms were installed with the aim to create an environment for relaxation for the children. The ceiling, roof and walls were dressed with soft cloth materials. Besides, it was made possible to damp the light of the room. By use of special equipment, lighting sceneries of stars, butterflies, clouds or fishes were exposed on the roof and walls. Sounds of whales, birds and soft music were delivered from loudspeakers on the walls (Lekolar, Sweden). The personnel were instructed to use the room in the afternoon or other periods when the children were getting tired and needed a short rest.

\section{Recovery room for the personnel}

At this school, a noise-isolated room was designed for relaxation and recovery of the personnel. The background noise of the room was below $40 \mathrm{dBA}$, and the room was equipped with a relaxing chair and a soft light. The employees at the two departments were instructed to use the room twice a day and not less than for $15 \mathrm{~min}$ at each occasion. One person per occasion was allowed to use the room. The usage of the room was controlled by the personnel who filled in a time schedule placed in the room.

\section{Regulation of the light}

In two departments at one preschool, the dining rooms, play halls and other strategic spaces used by the children were equipped with a possibility to damp the light. The original regulating system was changed into alternatives for electronic damping of the fluorescent lamps. At times for ending or lowering the playing activities of the children, for example in connection with rest, reading or other educational activities, the staff reduced the lighting of the spaces.

\section{SoundEar}

At one preschool, three SoundEar ${ }^{\circledR}$ (SoundEar A/S) were installed at each of the two participating departments. The sound ears were installed in the dining rooms, the play halls and dressing-rooms. The sound ears were calibrated to signal red at dBA levels above $70 \mathrm{dBA}$ and yellow at levels above $65 \mathrm{dBA}$. The personnel were instructed to use the SoundEar as an information source regarding the sound level at the departments. When the SoundEar signaled high noise level, the personnel were supposed to act and try to lower the sound level. The children were also educated about the meaning of the signals. They were repeatedly told to observe the signal and told to lower their voices at red signals.

\section{Noise and risk education}

At one preschool, two departments were given education in noise, noise exposure, health hazards and legislation, which was based on e-mail distribution of reading material, a library installed at the preschool and oral education at the departments for a period of 9 months. 


\section{Noise education for the children}

The measure was focused on teaching the children about sound, noise, hearing and hearing damages. This was carried out by an external pedagogue (AMMOT, Stockholm) and was based on entertaining and mixing the message into the text of songs. Besides, the children were engaged in indoor and outdoor activities to learn about what noise can be. The education by the pedagogue was followed up by the personnel at the departments who continued to implement the teaching material in the pedagogic course plan.

\section{Voice education for the personnel}

The measure was carried out by a legitimated speech therapist and focused on the use of the voices of the personnel. Among other things, the employees were instructed about how to use their voices when communicating with the children and other colleagues. They were also instructed about hazards of voice-related impairments and the health measures related to that.

\section{Statistical methods}

All statistical analyses were made using SPSS, version 17.0. Reliability analyses of the sound measurements for different weekdays and the creation of the KSD index were carried out using Cronbach's alpha. Differences in mean sound level and number of sound events before and after the implementation of the measures for measures were analyzed using paired samples $t$ test. Data regarding changes in subjective ratings of the sound environment and health were not normally distributed, and the changes in mean ratings were analyzed using the Wilcoxon rank-sum test. Group differences for changes in sound level and number of sound events above $85 \mathrm{dBA}$ were analyzed. ANCOVA, with the difference in mean number of children as a covariate, was used when analyzing changes in the sound level and mean number of sound events before and after implementation of the preventive measures. Group difference for acoustical and organizational measure regarding changes in subjective evaluations for the sound environment and different health variables was analyzed using one-way ANOVA. The impact of number of children on the sound level was analyzed using linear regression analyses. Level of significance was set to alpha $5 \%$ for all analyses.

\section{Ethics}

The study was approved by the regional ethical review board. All subjects in the study have been informed in written and verbal forms about the purpose of the study and its design. They were also informed that all data would be anonymous and only presented group-wise, that their participation was strictly voluntary and that they were allowed to end the participation at any time. All subjects gave their written agreement to participate.

\section{Results}

The results of the study are described in the perspective of the way in which the noise exposures were changed by use of the different types of measures and the way in which adverse effects were altered.

Presence of children at the departments

The number of children present at the departments during the weeks of measurements was controlled by use of the daily registrations made by the employees. Before the introduction of the preventive measures, an average of 13.5 (SD 2.4) children were present at the departments that underwent acoustical measures. During the second measurement, after the implementation of the measures, an average of 14.1 (SD 3.2) children were present. In the departments with organizational measures, an average of 14.0 (SD 2.4) children were present before the implementation of the measures. After the implementation, there were an average of 14.1 (SD 2.2) children. Similar registrations were made for the control preschools. An average of 14.7 (SD 3.7) children were present during the first study week and an average of 12.3 (SD 2.8) during the last study week.

\section{Reliability of the sound measurements}

The reliability of the sound measurements between the different working days was tested using Cronbach's alpha. All sound level measurements before and after the preventive measures had an Alpha score between 0.7 and 0.8.

Changes in noise levels

Table 1 is based on sound level data and mean number of sound events before the implementation of acoustical or organizational measures.

A mean of 25.0 (SD 8.1) whole-day noise dosimeter recordings were made at each preschool with acoustical measures. At each preschools with organizational measures, a mean of 24.6 (SD 4.0) whole-day noise dosimeter recordings were made. A mean of 22.7 (SD 4.0) whole-day noise dosimeter recordings were made at each control preschool.

Table 1 shows the mean values of the sound level before any measures had been made. As can be seen from the table, the equivalent noise levels ranged from 69 to 
Table 1 Descriptive data of mean values of the sound levels, separated by the different measures

\begin{tabular}{lclll}
\hline & $N$ & $\begin{array}{l}\text { dB(A)Leq } \\
\text { dosimeter } \\
\text { recording }\end{array}$ & $\begin{array}{l}\text { dB(A)Leq } \\
\text { stationary } \\
\text { recording }\end{array}$ & $\begin{array}{l}\text { Sound events } \\
\text { above } \\
85 \text { dB(A) }\end{array}$ \\
\hline New ventilation & 5 & 70.97 & 61.42 & \\
Sound absorbing tables & 4 & 71.17 & 66.08 & \\
Noise isolation wall panels & 6 & 69.43 & 61.45 & \\
New toys & 6 & 69.59 & 63.30 & 56.59 \\
Less children & 8 & 70.08 & 63.85 & 66.25 \\
New play hall & 4 & 71.33 & 64.80 & 55.59 \\
Acoustical measures & 33 & 70.29 & 63.40 & 93.26 \\
Voice education for the personnel & 5 & 71.36 & 64.03 & 153.92 \\
Noise pedagogics for the children & 3 & 68.71 & 62.31 & 121.65 \\
Recovery room for the personnel & 4 & 71.21 & 66.10 & 57.19 \\
Recovery room for the children & 4 & 71.46 & 64.70 & 79.35 \\
Regulation of the light & 6 & 70.05 & 64.94 & \\
Risk and noise education & 5 & 70.99 & 63.69 & 93.32 \\
SoundEar & 4 & 71.46 & 65.14 & 57.60 \\
Organizational measures & 31 & 70.8 & 64.29 & 64.44 \\
Control schools & 14 & 70.60 & &
\end{tabular}

$71 \mathrm{dBA}$, based on dosimeter recordings. The stationary sound levels ranged from 64 to $66 \mathrm{dBA}$. The number of sound events above $85 \mathrm{dBA}$ varied between 57 and 154 . Missing data regarding number of sound events above $85 \mathrm{dBA}$ depend on limitations in some of the noise dosimeters used.

Table 2 shows the differences in the sound levels before and after the introduction of the measures.

Paired samples $t$ tests showed significant changes when comparing the stationary noise levels of two preschools with acoustical measures and three preschools with organizational measures. No significant changes were seen for the control preschools.

Lower noise levels were seen for the preschool with new sound absorbing tables $t(4)=9.16, P=.001$, and the preschool with the new toys $t(5)=77.34, P=.001$. Improved stationary recorded sound levels were also seen for the preschool with risk and noise education $t(5)=3.33$, $P=.02$, recovery room for the personnel $t(3)=-.184$, $P=.001$, and recovery room for the children $t(3)=26.15$, $P=.001$.

No significant changes were seen for the noise dosimeter sound levels except for the preschool with the new lightning installed. At this preschool, the noise level increased after the implementation, $t(5)=-3.18, P=.03$. No significant changes were seen for the number of sound events above $85 \mathrm{dBA}$ for any of the evaluated measures.

\section{Changes in ratings of the sound environment}

Table 3 shows the means of the subjective evaluations of the sound environment before any measure had been taken. Rated sound level varied between 2.7 and 3.4 on the four-point graded scale. Rated sound fluctuation ranged from 4.7 to 6.3 on the seven-point graded scale, disturbance of the children's voices ranged from 3.0 to 3.8 , and noise from the children's activities ranged from 2.8 to 3.4 on the four-point graded scale. Noise annoyance varied between 40 and 74 on the $1-100 \mathrm{~mm}$ scale. Sound fatigue ranged from 2.3 to 4.8 on the six-point graded scale.

Significant subjective rated improvements were seen for the preschools with less children regarding disturbance from the children's voices $(z=-2.00, P=.046, r=$ $-0.45)$. The preschool with noise isolation wall panels rated an improvement regarding the sound fluctuation. This change, however, was not significant $(z=-1.84, P=.07$, $r=-0.53)$. Similar results were seen for the preschool with new toys $(z=-1.84, P=.07, r=-0.53)$.

No significant differences were seen for the subjective evaluations of the sound environment at preschools with organizational measures, except for preschool with voice education. At this preschool, the noise annoyance rating was higher after the implementation $(z=-2.02, P=.04$, $r=-0.64)$. The control preschools showed no significant difference in the subjective evaluations of the sound environment, except for an increased disturbance of noise from the children's activities $(z=-2.12, P=.03$, $r=-0.40$ ).

When analyzing differences group-wise, significant differences were seen for rated noise fluctuation among preschools with acoustical measures $(z=-2.39, P=.02$, $r=-0.29$ ), showing a lower rate of noise fluctuation (Table 4). 
Table 2 Changes in physical measurements after the introduction of the different measures

\begin{tabular}{|c|c|c|c|c|c|c|c|}
\hline & $N$ & $\begin{array}{l}\mathrm{dB}(\mathrm{A}) \text { Leq dosimeter } \\
\text { recording }\end{array}$ & $P$ & $\begin{array}{l}\mathrm{dB}(\mathrm{A}) \text { Leq stationary } \\
\text { recording }\end{array}$ & $P$ & $\begin{array}{l}\text { Sound events above } \\
85 \mathrm{~dB}(\mathrm{~A})\end{array}$ & $P$ \\
\hline New ventilation & 5 & .38 & .74 & -.38 & .89 & & \\
\hline Sound absorbing tables & 4 & -.71 & .33 & -1.18 & .01 & & \\
\hline Noise isolation wall panels & 6 & -.57 & .57 & 2.54 & .10 & & \\
\hline New toys & 6 & -.91 & .48 & -2.51 & .00 & & \\
\hline Less children & 8 & -.20 & .65 & -.12 & .82 & 7.97 & .63 \\
\hline New play hall & 4 & -.35 & .55 & -3.13 & .25 & -10.05 & .28 \\
\hline Acoustical measures & 33 & -.39 & .25 & -.60 & .33 & 10.87 & .41 \\
\hline Voice education for the personnel & 5 & -.79 & .30 & 2.12 & .06 & 24.49 & \\
\hline Noise pedagogics for the children & 3 & -.45 & .76 & -1.42 & .23 & & \\
\hline Recovery room for the personnel & 4 & .15 & .87 & -1.96 & .00 & -94.42 & .52 \\
\hline Recovery room for the children & 4 & .70 & .44 & -.52 & .00 & -45.15 & .06 \\
\hline Regulation of the light & 6 & 2.21 & .03 & 1.03 & .11 & 7.81 & .49 \\
\hline Risk and noise education & 5 & .05 & .95 & -.89 & .02 & -8.75 & .67 \\
\hline SoundEar & 4 & -.41 & .25 & -.07 & .94 & & \\
\hline Organizational measures & 31 & .32 & .32 & -.24 & .67 & -22.66 & .26 \\
\hline Control schools & 14 & -.07 & .87 & -.72 & .23 & .05 & 1.00 \\
\hline
\end{tabular}

Table 3 Descriptive data of mean values for the subjective evaluation of the sound measurements separated in types of different measures before the introduction of the preventive measures

\begin{tabular}{|c|c|c|c|c|c|c|c|}
\hline & $N$ & $\begin{array}{l}\text { Sound } \\
\text { level }\end{array}$ & $\begin{array}{l}\text { Sound } \\
\text { fluctuation }\end{array}$ & $\begin{array}{l}\text { Disturbance of } \\
\text { children's voices }\end{array}$ & $\begin{array}{l}\text { Disturbance of noise from } \\
\text { children's activities }\end{array}$ & $\begin{array}{l}\text { Noise } \\
\text { annoyance }\end{array}$ & $\begin{array}{l}\text { Sound } \\
\text { fatigue }\end{array}$ \\
\hline New ventilation & 5 & 2.67 & 4.67 & 3.00 & 2.83 & 40.00 & 3.00 \\
\hline Sound absorbing tables & 4 & 2.40 & 5.20 & 3.00 & 2.80 & 46.20 & 3.00 \\
\hline Noise isolation wall panels & 6 & 2.83 & 6.00 & 3.33 & 3.17 & 56.83 & 4.33 \\
\hline New toys & 6 & 2.83 & 6.20 & 3.17 & 3.00 & 57.40 & 3.17 \\
\hline Less children & 8 & 3.38 & 5.43 & 3.75 & 3.14 & 60.38 & 4.25 \\
\hline New play hall & 4 & 3.25 & 6.25 & 3.00 & 3.25 & 71.50 & 3.75 \\
\hline Acoustical measures & 33 & 2.91 & 5.58 & 3.26 & 3.14 & 54.94 & 3.65 \\
\hline Voice education for the personnel & 5 & 2.60 & 5.00 & 3.20 & 3.25 & 43.00 & 2.80 \\
\hline Noise pedagogics for the children & 3 & 3.00 & 4.75 & 3.25 & 3.00 & 47.75 & 2.25 \\
\hline Recovery room for the personnel & 4 & 3.50 & 4.75 & 3.50 & 2.75 & 65.25 & 3.75 \\
\hline Recovery room for the children & 4 & 2.75 & 5.75 & 3.50 & 3.00 & 61.25 & 4.50 \\
\hline Regulation of the light & 6 & 3.00 & 5.33 & 3.33 & 3.00 & 48.33 & 4.50 \\
\hline Risk and noise education & 5 & 3.40 & 6.20 & 3.40 & 3.40 & 71.80 & 4.00 \\
\hline SoundEar & 4 & 3.20 & 5.40 & 3.60 & 3.25 & 74.40 & 4.75 \\
\hline Organizational measures & 31 & 3.06 & 5.33 & 3.39 & 3.10 & 58.58 & 3.81 \\
\hline Control schools & 14 & 2.79 & 5.54 & 3.29 & 2.71 & 57.21 & 3.79 \\
\hline
\end{tabular}

Changes in experiences of stress at work, fatigue, effort-reward and cortisol

Table 5 shows descriptive data for different stress markers and different health effects before the introduction of the preventive measures. Rated stress ranged from 1.06 to 3.54 , SOFI from 1.13 to 1.94 , CAR from -3.94 to 10.67 and CDD from 12.22 to 21.17 .

As can be seen from Table 6, significant changes were observed for one of the tested variables. The preschool with 


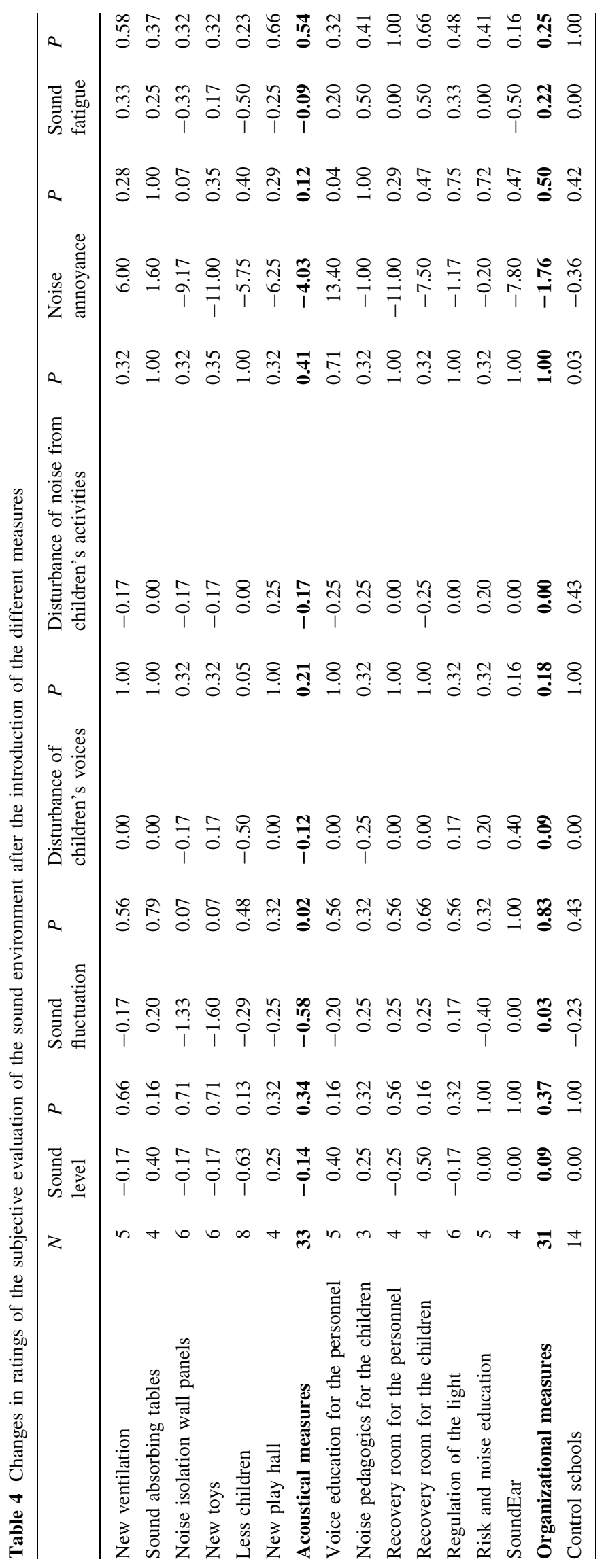


Table 5 Descriptive data of mean values for the subjective evaluation of stress, fatigue, effort-reward and cortisol before the implementation of the different measures

\begin{tabular}{|c|c|c|c|c|c|}
\hline & Stress at work & SOFI & ERI & CAR & CDD \\
\hline New ventilation & 3.21 & 1.13 & 0.44 & 1.63 & 18.60 \\
\hline Sound absorbing tables & 2.10 & 1.30 & 0.51 & -0.20 & 18.56 \\
\hline Noise isolation wall panels & 2.03 & 1.25 & 0.62 & 4.08 & 15.48 \\
\hline New toys & 1.97 & 1.13 & 0.49 & 6.50 & 12.22 \\
\hline Less children & 2.10 & 1.28 & 0.71 & 0.46 & 14.28 \\
\hline New play hall & 3.54 & 1.38 & 0.67 & -2.40 & 19.93 \\
\hline Acoustical measures & 2.37 & 1.24 & $\mathbf{0 . 5 8}$ & 1.87 & 15.99 \\
\hline Voice education for the personnel & 3.23 & 1.75 & 0.59 & -3.94 & 18.82 \\
\hline Noise pedagogics for the children & 1.06 & 1.25 & 0.49 & 10.67 & 18.30 \\
\hline Recovery room for the personnel & 2.75 & 1.38 & 0.67 & 1.45 & 19.83 \\
\hline Recovery room for the children & 2.75 & 1.56 & 0.67 & 4.87 & 21.17 \\
\hline Regulation of the light & 1.30 & 1.15 & 0.50 & -0.43 & 18.65 \\
\hline Risk and noise education & 2.17 & 1.44 & 0.85 & -3.68 & 15.98 \\
\hline SoundEar & 2.83 & 1.94 & 1.08 & 7.92 & 18.96 \\
\hline Organizational measures & 2.34 & 1.50 & 0.68 & 1.65 & 18.66 \\
\hline Control schools & 3.05 & 1.63 & 0.68 & 5.83 & 19.52 \\
\hline
\end{tabular}

Table 6 Changes in subjective stress, effort-reward, fatigue and cortisol after the introduction of the different measures

\begin{tabular}{|c|c|c|c|c|c|c|c|c|c|c|}
\hline & Stress at work & $P$ & ERI & $P$ & SOFI & $P$ & CAR & $P$ & CDD & $P$ \\
\hline New ventilation & -0.46 & 0.85 & -0.01 & 0.50 & -0.13 & 0.14 & 9.20 & 0.07 & 3.00 & 0.47 \\
\hline Sound absorbing tables & 0.80 & 0.07 & 0.01 & 0.72 & 0.25 & 0.35 & 2.58 & 0.69 & -2.32 & 0.59 \\
\hline Noise isolation wall panels & -0.03 & 1.00 & -0.02 & 0.34 & 0.69 & 0.29 & 6.23 & 0.46 & 8.18 & 0.08 \\
\hline New toys & -0.39 & 0.59 & -0.01 & 0.92 & 0.04 & 0.14 & 0.93 & 0.92 & 4.82 & 0.03 \\
\hline Less children & -0.37 & 0.17 & -0.10 & 0.12 & 0.06 & 0.08 & 4.96 & 0.14 & 3.43 & 0.26 \\
\hline New play hall & 0.29 & 0.20 & 0.13 & 0.14 & 1.19 & 0.27 & 0.30 & 1.00 & 0.00 & 1.00 \\
\hline Acoustical measures & -0.06 & 0.89 & -0.01 & 0.53 & 0.28 & 0.00 & 4.07 & 0.04 & 3.05 & 0.02 \\
\hline Voice education for the personnel & 0.04 & 1.00 & -0.06 & 0.35 & 1.45 & 0.18 & 4.28 & 0.35 & 4.40 & 0.14 \\
\hline Noise pedagogics for the children & 0.11 & 0.79 & 0.01 & 0.72 & 0.25 & 0.66 & -1.17 & 0.59 & -0.97 & 1.00 \\
\hline Recovery room for the personnel & -0.33 & 0.59 & 0.10 & 0.47 & 0.38 & 0.27 & -1.75 & 0.72 & -1.30 & 0.07 \\
\hline Recovery room for the children & -0.75 & 0.07 & -0.04 & 1.00 & -0.38 & 0.66 & 5.37 & 1.00 & 2.73 & 0.59 \\
\hline Regulation of the light & 1.30 & 0.04 & 0.00 & 0.75 & 0.80 & 0.03 & 10.78 & 0.03 & 0.67 & 0.60 \\
\hline Risk and noise education & -0.21 & 0.47 & 0.01 & 0.72 & 1.19 & 0.72 & 1.10 & 0.50 & 1.32 & 0.69 \\
\hline SoundEar & -0.50 & 0.14 & -0.39 & 0.07 & 0.87 & 0.47 & -3.02 & 0.69 & 0.24 & 0.69 \\
\hline Organizational measures & 0.00 & 0.85 & -0.05 & 0.08 & 0.70 & 0.53 & 2.65 & 0.17 & 1.09 & 0.60 \\
\hline Control schools & -0.04 & 0.68 & 0.05 & 0.16 & 0.48 & 0.76 & -3.36 & 0.31 & 2.32 & 0.18 \\
\hline
\end{tabular}

new toys showed significant increase in CDD $(z=-2.201$, $P=.03, r=-0.64)$ after use of the new toys.

No other significant differences were observed for any of the other acoustical measures. Analyzing all acoustical measures group-wise, significant improvements were seen for $\operatorname{CAR}(z=-2.09, P=.04, r=-0.25)$, CDD $(z=-1.84, P=.02, r=-0.28)$ and SOFI $(z=-3.06$, $P=.002, r=-0.39$ ).

Significant differences were also seen for different organizational measures. The preschool with a new light regulation system installed showed a significant increase in stress $(z=-2.03, P=.004, r=-0.59)$ as well as an 
Table 7 Descriptive data of mean values for the subjective evaluation of burnout, depression, sleep and sleep quality before the implementation of the different measures

\begin{tabular}{|c|c|c|c|c|c|}
\hline & Burnout & Depression & KSS before sleep & KSS after sleep & KSD index \\
\hline New ventilation & 2.24 & 5.50 & 6.53 & 5.38 & 3.82 \\
\hline Sound absorbing tables & 2.52 & 7.40 & 7.22 & 5.24 & 4.11 \\
\hline Noise isolation wall panels & 2.50 & 4.67 & 7.63 & 6.43 & 3.98 \\
\hline New toys & 2.98 & 6.33 & 6.76 & 6.68 & 3.65 \\
\hline Less children & 3.46 & 8.50 & 6.13 & 5.81 & 3.75 \\
\hline New play hall & 3.16 & 11.50 & 7.22 & 7.32 & 3.39 \\
\hline Acoustical measures & 2.83 & 7.14 & 6.85 & 6.08 & 3.79 \\
\hline Voice education for the personnel & 2.58 & 11.40 & 6.40 & 5.80 & 3.63 \\
\hline Noise pedagogics for the children & 2.64 & 9.00 & 6.53 & 5.98 & 3.78 \\
\hline Recovery room for the personnel & 2.94 & 7.50 & 6.80 & 6.10 & 3.72 \\
\hline Recovery room for the children & 3.96 & 13.25 & 6.26 & 7.21 & 3.81 \\
\hline Regulation of the light & 2.88 & 6.33 & 5.86 & 5.46 & 3.54 \\
\hline Risk and noise education & 3.41 & 10.20 & 6.91 & 5.88 & 3.73 \\
\hline SoundEar & 4.03 & 12.50 & 7.15 & 5.29 & 3.25 \\
\hline Organizational measures & 3.17 & 9.84 & 6.54 & 5.90 & 3.62 \\
\hline Control schools & 3.25 & 10.71 & 6.87 & 6.07 & 3.75 \\
\hline
\end{tabular}

Table 8 Descriptive data of mean values for the subjective evaluation of burnout, depression, sleep and sleep quality before the implementation of the different measures

\begin{tabular}{lrrrrrrrrrr}
\hline & Burnout & $P$ & Depression & $P$ & KSS before sleep & $P$ & KSS after sleep & $P$ & KSD index & $P$ \\
\hline New ventilation & 0.52 & 0.12 & -0.33 & 1.00 & -0.93 & 0.03 & 0.75 & 0.21 & -0.02 & 0.92 \\
Sound absorbing tables & 0.26 & 0.23 & 3.40 & 0.35 & -0.14 & 0.89 & 0.62 & 0.35 & -0.12 & 0.50 \\
Noise isolation wall panels & 0.00 & 0.92 & -0.83 & 0.72 & -0.19 & 0.75 & -1.03 & 0.25 & -0.04 & 0.83 \\
New toys & -0.04 & 0.92 & 2.83 & 0.68 & -0.04 & 0.89 & -1.08 & 0.23 & 0.41 & 0.12 \\
Less children & -0.27 & 0.58 & -0.38 & 0.89 & 0.29 & 0.78 & 0.08 & 0.61 & 0.16 & 0.26 \\
New play hall & 0.38 & 0.47 & 6.25 & 0.07 & -0.20 & 0.72 & -1.17 & 0.07 & 0.04 & 0.72 \\
Acoustical measures & $\mathbf{0 . 1 0}$ & $\mathbf{0 . 2 7}$ & $\mathbf{1 . 4 0}$ & $\mathbf{0 . 2 2}$ & $-\mathbf{0 . 1 8}$ & $\mathbf{0 . 3 2}$ & $-\mathbf{0 . 2 6}$ & $\mathbf{0 . 4 8}$ & $\mathbf{0 . 0 8}$ & $\mathbf{0 . 3 0}$ \\
Voice education for the personnel & 0.95 & 0.04 & -4.20 & 0.27 & 0.76 & 0.07 & 1.34 & 0.10 & 0.10 & 0.50 \\
Noise pedagogics for the children & 0.46 & 0.47 & -0.50 & 1.00 & 0.15 & 0.59 & -0.44 & 0.72 & -0.07 & 0.59 \\
Recovery room for the personnel & 0.33 & 0.47 & 2.00 & 0.36 & -0.46 & 0.72 & -1.79 & 0.07 & 0.07 & 0.47 \\
Recovery room for the children & 0.25 & 0.58 & -0.75 & 0.72 & -0.13 & 0.72 & -0.53 & 0.47 & -0.29 & 0.27 \\
Regulation of the light & 0.11 & 0.60 & 1.17 & 0.53 & -0.05 & 0.92 & 1.09 & 0.25 & 0.07 & 0.75 \\
Risk and noise education & -0.15 & 0.69 & -3.80 & 0.20 & 0.05 & 0.50 & 0.65 & 0.47 & 0.13 & 0.89 \\
SoundEar & -0.37 & 0.47 & -2.50 & 0.36 & 0.23 & 0.69 & 1.24 & 0.08 & 0.11 & 0.47 \\
Organizational measures & $\mathbf{0 . 2 3}$ & $\mathbf{0 . 1 0}$ & $-\mathbf{1 . 2 5}$ & $\mathbf{0 . 1 5}$ & $\mathbf{0 . 0 9}$ & $\mathbf{0 . 5 4}$ & $\mathbf{0 . 3 5}$ & $\mathbf{0 . 2 2}$ & $\mathbf{0 . 0 3}$ & $\mathbf{0 . 6 4}$ \\
Control schools & 0.28 & 0.12 & -0.21 & 0.76 & 0.39 & 0.29 & -0.02 & 0.98 & -0.07 & 0.53 \\
\hline
\end{tabular}

increased value of the SOFI rating $(z=-2.20, P=.03$, $r=-0.64)$ and a higher CAR value $(z=-2.20, P=.03$, $r=-0.64)$.

No significant group differences were observed at the reference preschools or the preschools with organizational measures.
Changes in experiences of burnout, depression sleepiness and sleep quality

Table 7 shows the descriptive data for burnout, depression, sleepiness and sleep quality before the introduction of the different measures. 
Table 9 Descriptive cortisol data of the different time points, separated by type of preventive measure

\begin{tabular}{llllllll}
\hline & \multicolumn{3}{l}{ Before } & & & After \\
\cline { 8 - 8 } \cline { 7 - 8 } & $N$ & Mean & SD & & $N$ & Mean & SD \\
\hline At wakeup & & & & & & \\
Acoustical & 35 & 13.6 & 6.7 & 36 & 13.6 & 7.5 \\
Organizational & 37 & 15.1 & 6.6 & 32 & 15.0 & 6.9 \\
Reference & 14 & 13.7 & 8.5 & 13 & 16.7 & 8.9 \\
1 h after wakeup & & & & & & \\
Acoustical & 35 & 15.2 & 6.3 & 36 & 19.5 & 7.1 \\
Organizational & 37 & 16.6 & 6.3 & 31 & 19.3 & 8.7 \\
Reference & 14 & 21.1 & 7.6 & 13 & 20.3 & 9.6 \\
11 a.m. & & & & & & \\
Acoustical & 35 & 3.4 & 2.2 & 36 & 3.8 & 3.1 \\
Organizational & 37 & 4.0 & 2.3 & 32 & 4.0 & 2.6 \\
Reference & 14 & 4.4 & 4.0 & 13 & 3.8 & 3.7 \\
9 p.m. & & & & & & \\
Acoustical & 35 & 1.2 & 1.3 & 36 & 1.4 & 1.2 \\
Organizational & 37 & 1.2 & 0.8 & 32 & 1.6 & 2.7 \\
Reference & 13 & 1.7 & 1.1 & 13 & 1.3 & 1.9 \\
\hline
\end{tabular}

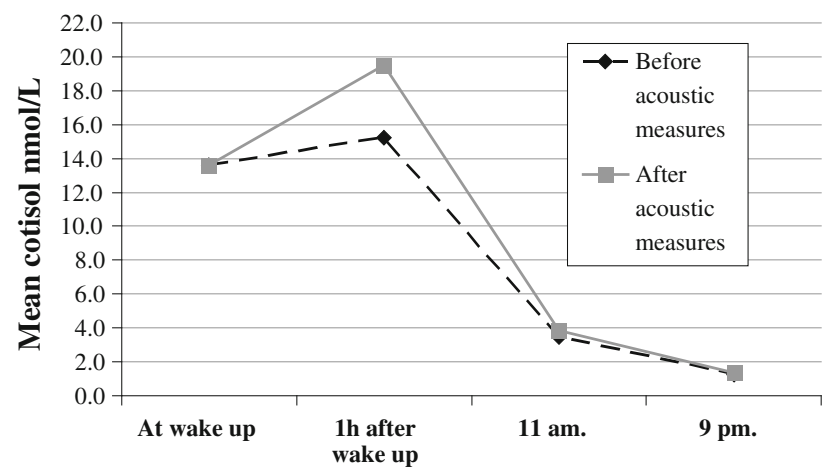

Fig. 1 Cortisol values at four different time points for preschools before and after the implementation of acoustical measures

As can be seen from Table 8, the preschool with the new ventilation system showed an improvement in KSS ratings before sleep, showing that they felt less tired in the evening after work $(\mathrm{z}=-.184, P=.03, r=-0.64)$. The preschool that underwent voice education showed a significant increase in burnout $(z=-2.02, P=.04, r=-0.64)$.

When analyzing differences groupwise, no significant differences in the effect were seen for the acoustical, organizational or the control preschools for any of the tested variables.

\section{Cortisol data}

Cortisol was collected at four different time points during one working day. Table 9 shows an overview of the data, showing the highest cortisol value $1 \mathrm{~h}$ after wakeup for all three types of preschools, separated by type of measures. The lowest value was obtained at 9:00 pm. Changes in cortisol values before and after the introduction of acoustical measures are also illustrated in Fig. 1.

Employees' ratings of the implemented measures

Figure 2 shows an overview of the mean ratings of the employees and whether an improvement in the sound level due to the implemented measure had occurred. In general, mean ratings of the overall improvement were higher for acoustical than for the organizational measures. Fewer children at the department were rated as the most beneficial of all tested measures.

Impact of number of children

The impact of the number of children on the acoustical environment is shown in Fig. 3.

As can be seen, there is a linear association between changes in the mean number of children present and changes in the sound level (mean values of the stationary sound levels before and after the use of the measures) using linear regression analyses $\left(r^{2}=.257, P<.01\right)$.

Similar results were seen for the associations between difference in mean number of children and dosimeter sound levels $\left(r^{2}=.053, P<.05\right)$ and the mean number of children and mean number of sound events above $85 \mathrm{dBA}$ $\left(r^{2}=.240, P<.05\right)$.

Acoustical and organizational measures and the effect on work environment and health compared to reference preschools

Since changes in the mean number of children and difference in the sound levels were correlated, mean number of children was entered as a covariate when analyzing changes in the physical sound measurements.

\section{Acoustical and organizational measures and the effect on the sound environment}

The ANCOVA of the differences in dosimeter sound levels between the two measurements with the mean number of children as a covariate revealed no statistically significant differences between the preschools with acoustical measures or organizational measures and the reference schools. The dosimeter sound level decreased at preschools with acoustical measures compared to the control group where the sound level increased. Preschools with organizational measures showed increased dosimeter sound levels similar to the control group, $F(2 / 74)=1.99, P=.15$. However, 
Fig. 2 Subjective ratings regarding improvements in the sound environment separated by types of measures $(1=$ no improvement, $2=$ small improvement, $3=$ a high improvement, $4=$ a very high improvement)

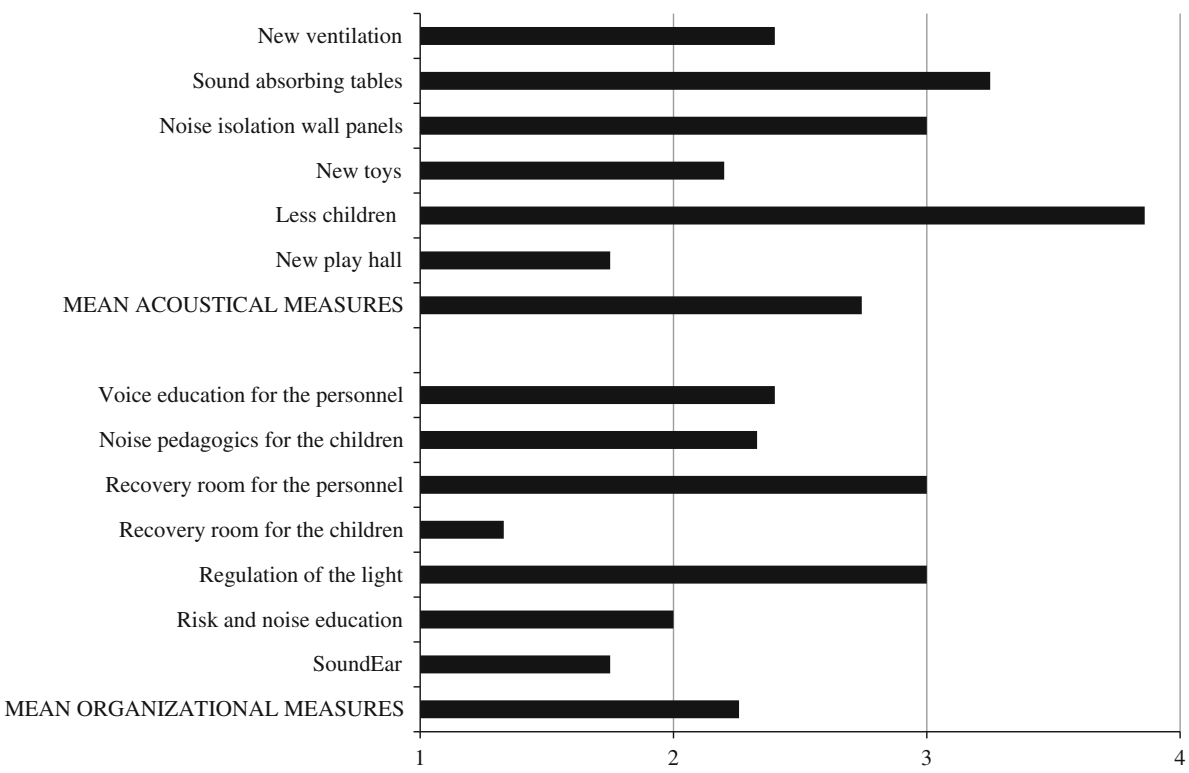

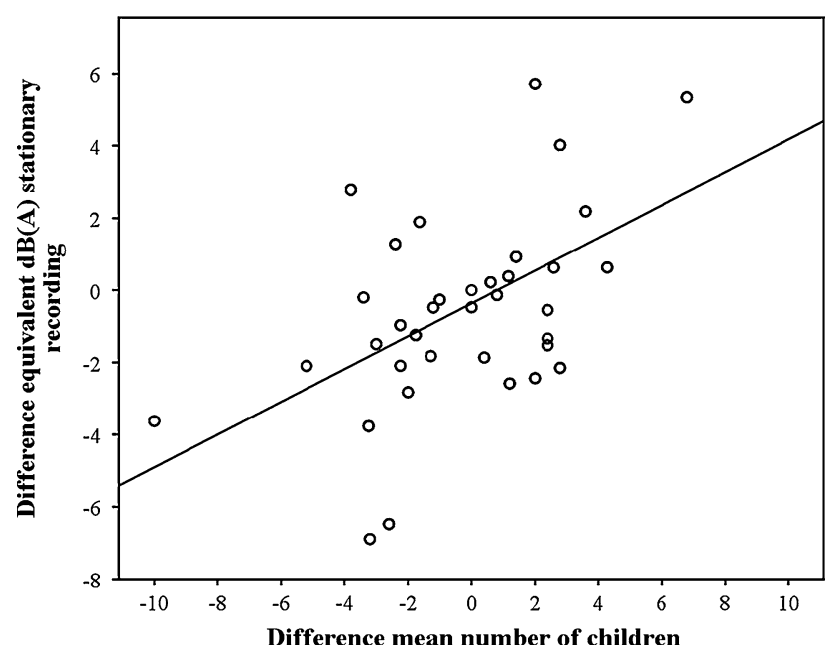

Fig. 3 Scatter plot showing linear regression between difference in mean number of children at the departments and its association with measured equivalent sound level

the difference in number children present at the departments during the measurement was significantly related to the sound level, $F(1 / 74)=5.66, P=.02$.

Similar results were seen for the stationary recordings, where the preschools with acoustical measures had lowered the sound levels more than preschools with organizational measures compared to the control preschools, $F(2 / 85)=$ $1.81, P=.17$. The difference in the number children present at the departments during the measurement was significantly related to the sound level, $F(1 / 85)=33.45$, $P=.001$.

Analyses also showed that preschools with preventive measures, both acoustical and organizational, had lowered the number of sound events above $85 \mathrm{dBA}$ compared to the reference preschools which showed an increase in the noise fluctuation. This difference, however, was not significant, $F(2 / 20)=.93, P=.41$. The difference in the number of children present at the departments during the measurement was significantly related to mean number of sound events above $85 \mathrm{dBA}, F(1 / 20)=5.86, P=.03$.

\section{Acoustical and organizational measures and the effect on subjectively experienced sound levels}

ANOVAs showed that personnel in the preschools with acoustical measures rated the sound level lower than before the introduction of the measures. Preschools with organizational measures and the reference schools both rated the sound levels as higher. The difference between the groups was not statically significant, $F(79 / 81)=.90, P=.41$.

Preschools with acoustic measures also reported an improvements in sound fluctuation compared to the control preschools. Preschools with organizational measures reported more sound fluctuation. This group difference was not significant, $F(76 / 78)=2.48, P=.09$.

Almost all preschools reported lower noise annoyance after use of the measures. Preschools with acoustical measures reported the largest improvement. The groups differences were, however, not significant, $F(78 / 80)=.33$, $P=.72$.

Preschools with acoustical measures reported lower disturbances regarding the children's voices after the introduction of the measures, whereas preschools with organizational measures and the control preschools rated more disturbances $F(79 / 81)=1.55, P=.22$.

Ratings regarding disturbing noise from the children's activities were improved in cases of acoustical measures, whereas no differences were reported for organizational measures. The control group reported increased disturbance. 
The group difference was significant when tested using ANOVAs, $(F(76 / 78)=3.46, P=0.04)$.

\section{Acoustical and organizational measures and the effect on subjectively rated stress and stress markers}

ANOVAs showed that stress ratings from personnel at the preschools with acoustical and organizational measures did not differ from those in the control preschools. Analyses regarding CAR showed that preschools with acoustical measures and organizational measures to a higher extent improved the CAR value, whereas the control schools showed a decrease in CAR value. The difference was, however, not significant, $F(74 / 76)=2.12, P=.13$.

All preschools showed an improvement in the CDD value. However, no group differences were seen, $F(73 / 75)=.44, P=.65$.

An improvement in the ERI value was seen for preschools both for acoustical and organizational measures compared to the control preschools. The difference was, however, not significant, $F(78 / 80)=1.17, P=.32$.

\section{Acoustical and organizational measures and the effect on rated health}

All preschools, including the control preschools, had higher burnout scores after the introduction of the measures. Preschools with acoustical measures had a lower increase in burnout, compared to preschools with organizational measures and the control schools. The group differences were, however, not significant, $F(78 / 80)=.34, P=.71$.

Positive changes, however not significant, was seen for depression in preschools with organizational measures, and for the control preschools, whereas preschools with acoustical measures had higher depression values, $F(78 / 80)=$ $1.31, P=.28$.

No improvements were seen for preschools with acoustical and organizational measures and the control group regarding the SOFI value (lack of energy), $F(67 / 69)=1.15$, $P=.32$.

Preschools with acoustical measures showed improvements in KSS before sleep compared to preschools with organizational measures and the control preschools which reported worsened KSS values $F(77 / 79)=.82, P=.45$.

Preschools with acoustical measures and the control preschools showed improvements in KSS after sleep compared to preschools with organizational measures which reported worsened KSS. These group differences were not significant, $F(79 / 81)=.81, P=.45$.

ANOVAs also revealed improved sleep quality using the KSD index for both the acoustical and organizational measures, whereas the control preschool showed an opposite effect. The group differences was, however, not significant $F(79 / 81)=.48, P=.62$.

\section{Discussion}

Significant lower sound levels were seen for the stationary recordings and the acoustical measures using sounddampened tables and new toys. Lower stationary levels by use of organizational measures were seen for recovery rooms for the personnel, recovery rooms for the children and the departments with noise education. In general, the reductions in noise levels after the use of the different measures were small and in most cases below the level of significance.

When considering the direction of the changes after introduction of the measures (dosimeter values, stationary values and sound levels events above $85 \mathrm{dBA}$ ), lower levels were seen for 11 of the $14(79 \%)$ analyzed effects of acoustical measures. Corresponding changes were seen for 11 of the $19(58 \%)$ analyzed effects of organizational measures. Thus in total, 22 of the $33(67 \%)$ analyzed effects of measures indicated a reduction in the noise levels. Despite the carefulness by which this conclusion should be drawn, the result is an indication of a better effect of acoustical than organizational measures.

The tendency seen for the group differences in the physical recorded changes in noise levels is supported by an equal change with respect to the employees' experiences of the sound environment. In total, 21 of the $36(58 \%)$ rated lower values (experienced noise levels, sound fluctuations, disturbance of the children's voices and activities, noise annoyance and sound fatigue) in the category of acoustical measures indicated a positive effect of the tested measures. Corresponding changes for category organizational measures were less. In fact, only 13 of 36 (36\%) rated values indicating a positive effect of these measures. As for the changes in noise levels, the changes in experiences of the employees were small and in most cases not significant. Changes in the subjective stress, effort-reward, fatigue and cortisol were even smaller. The same picture can be observed for the tested health effects: burnout, depression, sleepiness and sleep. The absence of effects on stress, fatigue and health effects reflects the low impact of each tested measure and also that these effects in several situations are effected by a large number of other factors than noise.

The observed small numerical changes in noise levels (dosimeter values as well as stationary levels) should be considered in the perspective of the changes in equivalent values and health hazards related to such changes. Based on the mathematical basics for noise levels and time of exposures, a reduction of $3 \mathrm{~dB}$ corresponds to an increase 
of $100 \%$ in exposure time, maintaining the same risk, a reduction of $1.5 \mathrm{~dB}$ to a $50 \%$ increase, and $0.75 \mathrm{~dB}$ reduction to a $25 \%$ increase in exposure time. The observed changes in dosimeter noise levels, between 0.05 and $0.95 \mathrm{~dB}$ for the tested acoustical measures and between 0.07 and $2.51 \mathrm{~dB}$ for the organizational tested measures, might at least in some cases have an impact on the hazards of the noise exposure. Besides, in several cases, the effects of the measures are not only to be find in a lowering of the dBA-value, but rather an improvement of the sound quality. A well-grounded assumption would be that the positive effects of several of the tested measures are combinations between lower levels and better sound climate. If so, combining several of the tested measures would be a successful concept.

The effect of the number of children on the noise levels showed an expected lowering of the noise levels by reducing the number of children at the departments. Analyzing all departments, changes in noise level and its relation to changes in number of children, significant positive effects were seen for the stationary as well as personal noise recordings. A decrease in the number of children at the departments also reduced the number of sound events above $85 \mathrm{dBA}$. Considering the children as static noise sources, removing half of the children at each department should result in a change of $3 \mathrm{dBA}$. In this study, a mean of 14 children were present at the departments during both periods of sound measurements. A reduction by half of the children $(N=7)$ in this study showed a reduction close to $4 \mathrm{~dB}$. The deviation by about $1 \mathrm{~dB}$ from the expected reduction can probably be explained by the Lombard effect verified in several previous studies (Junqua 1993). Noteworthy is, however, that our results is based on the differences between pre- and post-measurements, where the sound environment has been altered with different measures between the measurements. As a result, there is a risk that we may underestimate the Lombard effect due to reduced sound levels post-measurement.

Significant effects, groupwise, were only seen for the rated noise fluctuation. A specific aim of the study was to see whether an improvement in the sound environment either objectively or subjectively could have an impact on the personnel's health. As previously mentioned, the noise effects of health were small and in most cases absent or not significant. Analyzing changes in CAR and CDD, however, showed that departments with acoustical measures had a significant positive change compared to the organizational and control group showing no improvements. Employees at departments with acoustical measures also rated their fatigue significantly lower after the introduction of the measures.

A well-grounded assumption would be that the effects of the organizational types of measures are strongly affected by the reduced possibility of the personnel to support the daily environmental work to improve the noise situation at the departments. The effects of the organizational measures probably are strongly reduced due to the already stressful work with lack of time for participating in the preventive work. The conflict between support to the environmental work and duties in the education and care taking of the children are of course less pronounced and often nonpresent in the cases of acoustical measures. The acoustical measures need less involvement of the personnel, whereas the organizational measures are highly dependent on the personnel's commitment and the work they put into working with the measure. The effects of organizational measures in most cases also must be considered to take longer time to be implemented in changes of the physical environment and thus effects on the employees. Education to the children or personnel and changes related to the organization of the work are normally much more timeconsuming processes than measures related to changing wall materials, tables, toys or technical equipment at the departments.

A weakness of this study is the rather small number of observations for each evaluated measure. As a result, the statistical power will be low. Even using a control group to adjust for time-related changes, confounders beyond control might have affected the outcome of the study, some tested measures probably being affected more than others. The use of more preschools, departments and employees were discussed when designing the study. Increasing the power of the study, however, was in conflict with time needed for such a study. Increasing the study group two, three or four times were considered to be in conflict with making repeated measures over 3-5 years. Loss of subjects and the risk for irrelevant changes over time were considered to lower the reliability of the study. The analyses using the employees as their own controls were considered of great importance and also to limit the period of the study. All participants were aware of the purpose of the study and were also informed about the actual measure introduced at their department. This may have influenced the participants and biased their evaluations of the different measures due to their expectations of the introduced measure. The possibility of making a blinded study and thus avoiding this bias in the subjective evaluations of the different measures was not possible since the implementation of the preventive measure to a high extent relies on the cooperation and permission from the personnel. Controlling for this by use of placebo measures at other preschools was not possible due to the nature of the preventive measures evaluated in the study. The risk of bias due to expectations of the introduced measures was minimized by the study design. The participants evaluated the sound environment before and after the introduction of each 
measure with 1 year between the two ratings. The measures were also evaluated by use of objective sound measurements. Figure 2 in the article is only based on ratings for each specific measure after the introduction and may thus be biased by the participant's expectations. However, these data are not used in any analyses and are only used as descriptive data.

The control preschools were informed about the purpose of the study and their roles in the project. Preferably in a controlled experimental situation, they would not have been informed about this. However, we considered it of importance to maintain control of the occupational environment and to avoid physical or organizational changes similar to the tested measures. High transparency was part of the study design and beneficial for the reliability of the study. Control regarding the implementation of the tested measures outweighed the disadvantages of the possible expectations from the participants.

Future studies are suggested to include several acoustical measures combined by deeper control of the non-workrelated variables that might interact with the noise-induced effects.

Acknowledgments This study was supported by AFA Insurance, Stockholm Sweden (Grant number: 070117).

Conflict of interest The authors declare that they have no conflict of interest.

\section{References}

Åhsberg E (2000) Dimensions of fatigue in different working populations. Scand J Psychol 41(3):231-241. doi:10.1111/14679450.00192

Åhsberg E, Gamberale F, Kjellberg A (1997) Perceived quality of fatigue during different occupational tasks development of a questionnaire. Int J Ind Ergon 20:121-135

Akerstedt T, Hume K, Minors D, Waterhouse J (1994) The subjective meaning of good sleep, an intraindividual approach using the Karolinska Sleep Diary. Percept Mot Skills 79(1 Pt 1):287-296

Bech P, Rasmussen NA, Olsen LR, Noerholm V, Abildgaard W (2001) The sensitivity and specificity of the major depression inventory, using the present state examination as the index of diagnostic validity. J Affect Disord 66(2-3):159-164. doi: 10.1016/s0165-0327(00)00309-8

Bies DA, Hansen CH (2009) Engineering noise control, 3rd edn. Taylor \& Francis Ltd, London

Clow A, Thorn L, Evans P, Hucklebridge F (2004) The awakening cortisol response: methodological issues and significance. Stress 7(1):29-37. doi:10.1080/10253890410001667205

Eklöf M, Ingelgård A, Hagberg M (2004) Is participative ergonomics associated with better working environment and health? A study among Swedish white-collar VDU users. Int J Ind Ergon 34(5):355-366. doi:10.1016/j.ergon.2004.04.013
Grossi G, Perski A, Evengard B, Blomkvist V, Orth-Gomer K (2003) Physiological correlates of burnout among women. J Psychosom Res 55(4):309-316

Hansen HC (2005) Noise control. Taylor \& Francis Ltd, London

Junqua J (1993) The Lombard reflex and its role on human listeners and automatic speech recognizers. J Acoust Soc Am 93(1):510

Kjellberg A, Wadman C (2002) Subjektiv stress och dess samband med psykosociala förhållanden och besvär. En prövning av Stress-Energi-modellen. Arbete och Hälsa 12:31

Kudielka BM, Wust S (2010) Human models in acute and chronic stress: assessing determinants of individual hypothalamuspituitary-adrenal axis activity and reactivity. Stress 13(1):1-14. doi:10.3109/10253890902874913

Lindeberg SI, Ostergren PO, Lindbladh E (2006) Exhaustion is differentiable from depression and anxiety: evidence provided by the SF-36 vitality scale. Stress 9(2):117-123. doi:10.1080/10253 890600823485

Melamed S, Kushnir T, Shirom A (1992) Burnout and risk factors for cardiovascular diseases. Behav Med 18(2):53-60

Melamed S, Ugarten U, Shirom A, Kahana L, Lerman Y, Froom P (1999) Chronic burnout, somatic arousal and elevated salivary cortisol levels. J Psychosom Res 46(6):591-598

Norlander T, Johansson $\AA$, Bood S (2005) The affective personality: its relation to quality of sleep, well-being and stress. Soc Behav Pers Int J 33(7):709-722

Olsen LR, Jensen DV, Noerholm V, Martiny K, Bech P (2003) The internal and external validity of the major depression inventory in measuring severity of depressive states. Psychol Med 33(02):351-356. doi:10.1017/S0033291702006724

Orion D (2010) Spectria cortisol RIA coated tube radioimmunoassay. Cat NO 06119

Persson R, Garde AH, Hansen ÅM, Ørbæk P, Ohlsson K (2003) The influence of production systems on self-reported arousal, sleepiness, physical exertion and fatigue-consequences of increasing mechanization. Stress Health 19(3):163-171. doi:10.1002/smi.967

Shirom A, Westman M, Shamai O, Carel RS (1997) Effects of work overload and burnout on cholesterol and triglycerides levels: the moderating effects of emotional reactivity among male and female employees. J Occup Health Psychol 2(4):275-288

Siegrist J (1996) Adverse health effects of high-effort/low-reward conditions. J Occup Health Psychol 1(1):27-41

Sjodin F, Kjellberg A, Knutsson A, Landstrom U, Lindberg L (2012a) Noise and stress effects on preschool personnel. Noise Health 14(59):166-178

Sjodin F, Kjellberg A, Knutsson A, Landstrom U, Lindberg L (2012b) Noise exposure and auditory effects on preschool personnel. Noise Health 14(57):72-82. doi:10.4103/1463-1741.95135

Smith BJ (1996) Acoustics and noise control, 2nd edn. Longman, Harlow

Snyder DS (2000) Active noise control primer. Springer, New York

Swedish Work Environment Authority (2010) Work-related disorders. Work Environment Statistics, vol 4

Wadman C, Kjellberg A (2007) The role of the affective stress response as a mediator for the effect of psychosocial risk factors on musculoskeletal complaints-part 2: hospital workers. Int J Ind Ergon 37(5):395-403. doi:10.1016/j.ergon.2006.12.001

WHO (1993) ICD-10 classification of mental and behavioral disorders. Diagnostic criteria for research. World Health Organization, Geneva

Wüst S, Wolf J, Hellhammer DH, Federenko I, Schommer N, Kirschbaum C (2000) The cortisol awakening response-normal values and confounds. Noise Health 7:78-88 